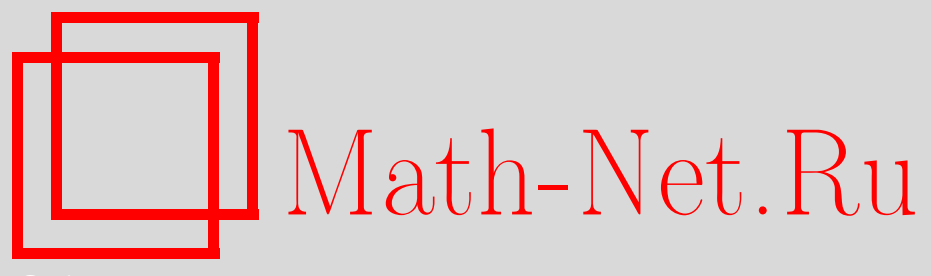

С. А. Степин, Оценки ядра и регуляризованный след полугруппы, порожденной потенциальным возмущением билапласиана, УМH, 2011, том 66, выпуск 3, 205-206

DOI: https://doi.org/10.4213/rm9431

Использование Общероссийского математического портала Math-Net.Ru подразумевает, что вы прочитали и согласны с пользовательским соглашением http: //www. mathnet.ru/rus/agreement

Параметры загрузки:

IP: 54.80 .97 .219

26 апреля 2023 г., $15: 46: 29$

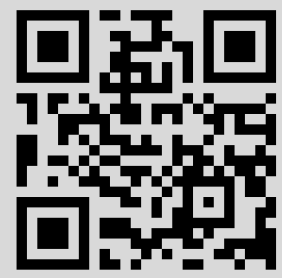




\section{Оценки ядра и регуляризованный след полугруппы, порожденной потенциальным возмущением билапласиана}

\section{С. А. Степин}

Цель сообщения - представить асимптотические формулы и оценки для интегрального ядра $G_{V}(x, y, t)$ полугруппы $U(t)=\exp \left(t H_{V}\right)$ с генератором $H_{V}=-P(i \nabla)+V(x)$, где $P(\xi)=|\xi|^{4} / 4$ и $V(x) \in \mathrm{L}_{1}\left(\mathbb{R}^{3}\right)$. Установленные оценки и способ их получения распространяются на случай положительно определенной формы $P(\xi)$ и ограниченного интегрируемого потенциала в пространстве произвольного числа измерений.

Поведение фундаментальных решений параболических уравнений (функций Грина) при $t \downarrow 0$ исследовалось в ряде публикаций, по большей части относящихся к случаю уравнений диффузионного типа, когда для этой цели применима вероятностная техника. С предметом настоящей заметки непосредственно связаны работы [1]-[5], где акцент делается на изучение эволюционных полугрупп, порожденных эллиптическими операторами порядка выше второго. На рассматриваемой здесь модельной задаче проявляются характерные особенности, составляющие существенное отличие от случая диффузионных полугрупп (см. [6], [7]).

Интегральное ядро $G_{0}(x, y, t)$ невозмущенной полугруппы $U_{0}(t)=\exp \left(-t \Delta^{2} / 4\right)$ имеет вид $(2 \pi)^{-3} \int \exp \left(-t|\xi|^{4} / 4+i(x-y, \xi)\right) d \xi$ и допускает оценку

$$
\left|G_{0}(x, y, t)\right| \leqslant C t^{-3 / 4} \exp \left(-\frac{1}{4} \frac{|x-y|^{4 / 3}}{t^{1 / 3}}\right), \quad C=(2 \pi)^{-3} \int e^{-P(\xi) / 10} d \xi .
$$

Метод перевала позволяет (см. [2], [3]) получить асимптотическую формулу

$$
\begin{aligned}
G_{0}(x, y, t)= & \frac{\pi^{-3 / 2}}{\sqrt{6}} \frac{t^{-1 / 2}}{|x-y|} \exp \left(-\frac{3}{8} \frac{|x-y|^{4 / 3}}{t^{1 / 3}}\right) \\
& \times\left\{\sin \left(\frac{3 \sqrt{3}}{8} \frac{|x-y|^{4 / 3}}{t^{1 / 3}}\right)+O\left(\frac{t^{1 / 3}}{|x-y|^{4 / 3}}\right)\right\} .
\end{aligned}
$$

Полугруппа $U(t)$ представима рядом теории возмущений

$$
U(t)=\sum_{n=0}^{\infty} U_{n}(t), \quad U_{n}(t)=\int_{0}^{t} U_{0}(s) V U_{n-1}(t-s) d s,
$$

фактически совпадающим с представлением параметрикса фундаментального решения $G_{V}(x, y, t)$ соответствующего параболического уравнения. Применительно к уравнениям диффузионного типа этот подход приводит (см. [6]) к известной формуле Фейнмана-Каца.

Теорема 1. Если потенциал $V \in \mathrm{C}^{2}\left(\mathbb{R}^{3}\right) \cap \mathrm{L}_{1}\left(\mathbb{R}^{3}\right)$ ограничен, то

$$
G_{V}(x, x, t)=(2 \pi)^{-3} t^{-3 / 4}(1+t V(x)) \int e^{-P(\xi)} d \xi+O(\sqrt{t}), \quad t \downarrow 0,
$$

а при фиксированных $x \neq y$ и достаточно мальх $t>0$ справедлива формула

$G_{V}(x, y, t)=G_{0}(x, y, t)\left(1+t \int_{0}^{1} V(x+(y-x) \tau) d \tau\right)+O\left(t^{7 / 12} \exp \left(-\frac{3}{8} \frac{|x-y|^{4 / 3}}{t^{1 / 3}}\right)\right)$.

Способ отыскания данных формул идейно родственен вероятностному подходу, используемому при вычислении коэффициентов коротковременного разложения теплового ядра, основанного на его представлении в виде винеровского интеграла (см. [7]). 
Несмотря на то что в данной ситуации ядро $G_{0}(x, y, t)$ невозмущенной полугруппы $U_{0}(t)$ не является плотностью вероятности перехода марковского процесса, оно обладает рядом свойств переходной плотности. Так, соотношение

$$
\int G_{0}(x, z, s) z G_{0}(z, y, t-s) d z=G_{0}(x, y, t)\left(x+\frac{(y-x) s}{t}\right)
$$

представляет собой аналог формулы для математического ожидания положения броуновской частицы $b(s)$, если $b(0)=x$ и $b(t)=y$. Указанная формула играет важную роль в предложенной конструкции, включающей оценку погрешности борновского приближения функции Грина и нахождение асимптотики при $t \downarrow 0$ первой поправки теории возмущений $\int_{0}^{t} d s \int G_{0}(x, z, s) V(z) G_{0}(z, y, t-s) d z$.

Теорема 2. В предположении ограниченности потенциала $V \in \mathrm{L}_{1}\left(\mathbb{R}^{3}\right)$ функция Грина $G_{V}(x, y, t)$ непреръвна и справедливо неравенство

$$
\left|G_{V}(x, y, t)\right| \leqslant C t^{-3 / 4} \exp \left(-\frac{1}{4} \frac{|x-y|^{4 / 3}}{t^{1 / 3}}\right) \sum_{n=1}^{\infty} \frac{\Gamma(1 / 4)^{n}}{\Gamma(n / 4)}\left(C t^{1 / 4} \int|V(x)| d x\right)^{n-1},
$$

оператор $U(t)-U_{0}(t)-$ ядерный в $\mathrm{L}_{2}\left(\mathbb{R}^{3}\right) u$

$$
\operatorname{Tr}\left(U(t)-U_{0}(t)\right)=(2 \pi)^{-3} t^{1 / 4} \int e^{-P(\xi)} d \xi \int V(x) d x+O(\sqrt{t}), \quad t \downarrow 0 .
$$

Отметим, что регуляризованный след в борновском приближении совпадает с главным членом выписанной асимптотики.

\section{Список литературы}

[1] М. В. Федорюк, Матем. сб., 62:4 (1963), 397-468. [2] М. А. Евграфов, М. М. Постников, Матем. сб., 82:1 (1970), 3-29; англ. пер.: М. A. Evgrafov, M. M. Postnikov, Sb. Math., 11:1 (1970), 1-24. [3] С. Г. Гиндикин, М. В. Федорюк, Матем. сб., 91:4 (1973), 500-522; англ. пер.: S. G. Gindikin, M. V. Fedorjuk, Sb. Math., 20:4 (1973), 519-542. [4] M. А. Евграфов, Матем. сб., 112:3 (1980), 331-353; англ. пер.: М. A. Evgrafov, Math. USSR-Sb., 40:3 (1981), 305-324. [5] М. М. Постников, Дифференииальные уравнения и динамические системы, Сборник статей. К 80-летию со дня рождения академика Евгения Фроловича Мищенко, Тр. МИАН, 236, Наука, М., 2002, 272-284; англ. пер.: М. M. Postnikov, Proc. Steklov Inst. Math., 236 (2002), 260-272. [6] С. А. Степин, Докл. AH, 420:4 (2008), 459-462; англ. пер.: S. A. Stepin, Dokl. Math., 77:3 (2008), 424-427. [7] С. А. Степин, Дифференциалъные уравнения и топология. II, Сборник статей. К 100-летию со дня рождения академика Льва Семеновича Понтрягина, Тр. МИАН, 271, МАИК, М., 2010, 241-258; англ. пер.: S. А. Stepin, Proc. Steklov Inst. Math., 271 (2010), 228-245.

\section{C. А. Степин (S. A. Stepin)}

Московский государственный университет им. М. В. Ломоносова;

Institute of Mathematics, University of Bialystok
Представлено Р. А. Минлосом Принято редколлегией 03.04.2011 